\title{
Prognostic risk factors for respiratory failure after esophagectomy
}

\author{
Quanguan Su\#, Huan Li", Honghong Yan, Wenxiao Wei, Wei Liao, Gang Ma \\ Sun Yat-sen University Cancer Center, State Key Laboratory of Oncology in South China, Collaborative Innovation Center for Cancer Medicine, \\ Guangzhou, China \\ Contributions: (I) Conception and design: G Ma, Q Su, W Liao; (II) Administrative support: G Ma; (III) Provision of study materials or patients: \\ Q Su, H Li, W Liao; (IV) Collection and assembly of data: Q Su, H Li, H Yan; (V) Data analysis and interpretation: Q Su, H Li, W Wei; (VI) \\ Manuscript writing: All authors; (VII) Final approval of manuscript: All authors. \\ \#These authors contributed equally to this work. \\ Correspondence to: Gang Ma; Wei Liao. Sun Yat-sen University Cancer Center, State Key Laboratory of Oncology in South China, Collaborative \\ Innovation Center for Cancer Medicine, Guangzhou, China. Email: magang@sysucc.org.cn; liaowei@sysucc.org.cn.
}

Background: Esophagectomy is a high-risk surgical procedure with significant postoperative morbidity and mortality. Respiratory failure is one of the most common complications after esophageal cancer surgery. The purpose of this study was to explore risk factors that affect the recovery of respiratory failure in this kind of patients.

Methods: Totally 2,360 patients underwent esophagectomies with cervical anastomosis or intrathoracic anastomosis in our center from January 2012 to December 2017. Among them, 130 patients with respiratory failure requiring mechanical ventilation were included in the study. The Spearman's rank correlation test and Mann-Whitney $\mathrm{U}$ test were performed to identify the influential factors for the duration of mechanical ventilation.

Results: Most patients (81.5\%) in this study recovered in the first two weeks of mechanical ventilation, the average duration of mechanical ventilation was 10.1 days. Statistical analysis indicated that lung function test parameters such as $\mathrm{FEV}_{1} / \mathrm{FVC}$ ratio and $\mathrm{RV} / \mathrm{TLC}$ ratio could have significant influence on the duration of mechanical ventilation. The abnormally increased $\mathrm{FEV}_{1} / \mathrm{FVC}$ ratio and RV/TLC ratio could obviously prolong the ventilation duration. Old age, thrombocytopenia, the elevated creatinine, C-reactive protein (CRP) and lactate could also significantly lengthen the duration of mechanical ventilation.

Conclusions: Abnormally increased $\mathrm{FEV}_{1} / \mathrm{FVC}$ ratio and RV/TLC ratio as well as old age, thrombocytopenia, elevated creatinine, CRP and blood lactate were found to be risk factors for the recovery of patients with respiratory failure after esophagectomies.

Keywords: Prognostic risk factors; respiratory failure; mechanical ventilation; esophagectomy

Submitted Mar 04, 2020. Accepted for publication Sep 02, 2020.

doi: 10.21037/tcr-20-1326a

View this article at: http://dx.doi.org/10.21037/tcr-20-1326a

\section{Introduction}

Esophageal cancer is a common malignant tumor worldwide (1), and surgery is the most important treatment for early and middle stage esophageal cancer. Although the surgical techniques have made remarkable progress in recent years (2-4), esophagectomy remains a high-risk operation with many postoperative complications $(5,6)$.

Respiratory failure is a major complication after esophagectomy, it has gradually become the main cause of postoperative death in esophageal cancer (7). The most common causes of respiratory failure are acute respiratory distress syndrome (ARDS) and pneumonia. There are many factors leading to respiratory complications after esophageal cancer surgery, such as long-term anesthesia, body cavity opening, intraoperative bleeding, limited expansion of lung tissue, impaired diaphragm function, postoperative pain, coughing weakness, etc. All these factors can impair the 
respiratory function and lead to respiratory failure (8).

Respiratory failure directly extends the length of ICU stay and total hospital stay, increases hospitalization costs, influences the recovery process and even leads to death of patients. But so far, there have been few reports on the prognostic factors of postoperative respiratory failure in esophageal cancer.

Therefore, the purpose of this study was to record the recovery process of patients with respiratory failure and tracheal intubation after esophagectomies, and to analyze the impacts of different factors on patients' recovery duration. This may help to provide some meaningful reference for the treatment of patients.

We present the following article in accordance with the STROBE reporting checklist (available at http://dx.doi. org/10.21037/tcr-20-1326a).

\section{Methods}

The study was conducted in accordance with the Declaration of Helsinki (as revised in 2013). The study was approved by Ethics Committee of Sun Yat-sen University Cancer Center of B2019-028-01 and informed consent was taken from all the patients.

In total, 2,360 patients underwent esophagectomies with cervical anastomosis or intrathoracic anastomosis from January 2012 to December 2017 in the thoracic surgery department of Sun Yat-sen University Cancer Center. Surgical methods were selected according to tumor location and other factors. In our institute, all cervical anastomosis patients underwent McKeown esophagectomies. And most of the intrathoracic anastomosis patients underwent left transthoracic procedures, only a few of them underwent Ivor-Lewis esophagectomies.

We included all the patients who suffered respiratory failure and underwent mechanical ventilation after esophageal cancer resection. Respiratory failure refers to the severe impairment of pulmonary ventilation caused by various causes, which makes it difficult to maintain adequate gas exchange at rest, resulting in hypoxemia with (or without) hypercapnia. Under the condition of breathing air, when the blood gas analysis examination shows that the arterial blood oxygen partial pressure $\left(\mathrm{PaO}_{2}\right)<60 \mathrm{mmHg}$, with or without carbon dioxide partial pressure $\left(\mathrm{PaCO}_{2}\right)$ $>50 \mathrm{mmHg}$, it can be diagnosed as respiratory failure. This study focused on patients with respiratory failure who required mechanical ventilation. So, patients enrolled in this study could be regarded as "Respiratory Failure Requiring
Invasive Mechanical Ventilation". For cases with mechanical ventilation less than 48 hours, it can be considered as a transitional mechanical ventilation after surgical anesthesia, which is not included in the scope of this study.

After a period of mechanical ventilation, these patients were successfully extubated and transferred out of the ICU. In order to make more accurate evaluation about the impact of different factors on the rehabilitation of respiratory failure, we excluded patients who died in hospital due to various reasons like massive hemorrhage, cardiac arrest, massive cerebral infarction, etc.

So finally, a total of 130 patients were included in the study. Relevant clinical and pathological data, including lung function test outcomes were obtained from medical records of each patient. As we know that the inflammatory response usually reaches its peak within one week, we collected the peak values of white blood cell (WBC) count, serum creatinine, C-reactive protein (CRP) and lactate during the first week after mechanical ventilation. Postoperative information such as endotracheal intubation duration, length of ICU stay and total hospital stay and treatment outcome was also collected.

\section{Statistical analytics}

Continuous variables were summarized by mean and SD. Frequencies and percentages were used to express categorical variables. The Spearman's rank correlation test and Mann-Whitney $U$ test were performed to identify the influential factors for the duration of tracheal intubation. A probability of less than 0.05 was considered statistically significant. Statistical analysis was performed by using SPSS software (version 21) for Windows $\left(\right.$ SPSS $^{\circledR}$ Inc., Chicago, IL, USA).

\section{Results}

In total, 2,360 esophageal cancer patients underwent esophagectomies at our institute from January 2012 to December 2017. Among them, 130 patients were eligible to enter the study. As shown in Figure 1, the duration of mechanical ventilation for patients in this study ranged from 3 to 74 days. The average duration was 10.1 days. Seventy-one $(54.6 \%)$ patients recovered within one week after tracheal intubation. Another 35 cases $(26.9 \%)$ recovered successfully in the second week. It can be seen that most patients $(81.5 \%)$ recovered in the first two weeks of mechanical ventilation. 


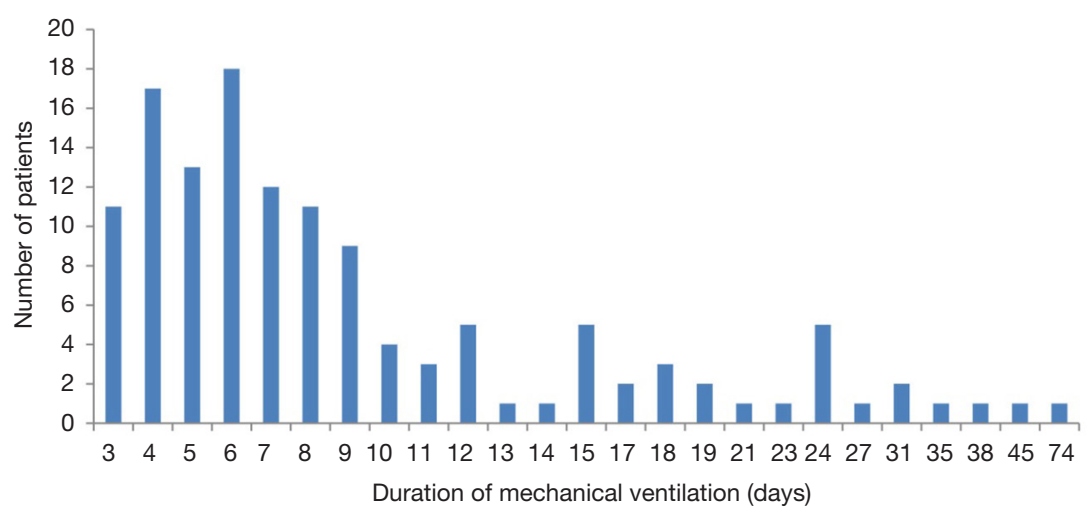

Figure 1 Distribution of mechanical ventilation duration.

Table 1 Clinical characteristics and lung function of the patients and their relationship with the duration of mechanical ventilation

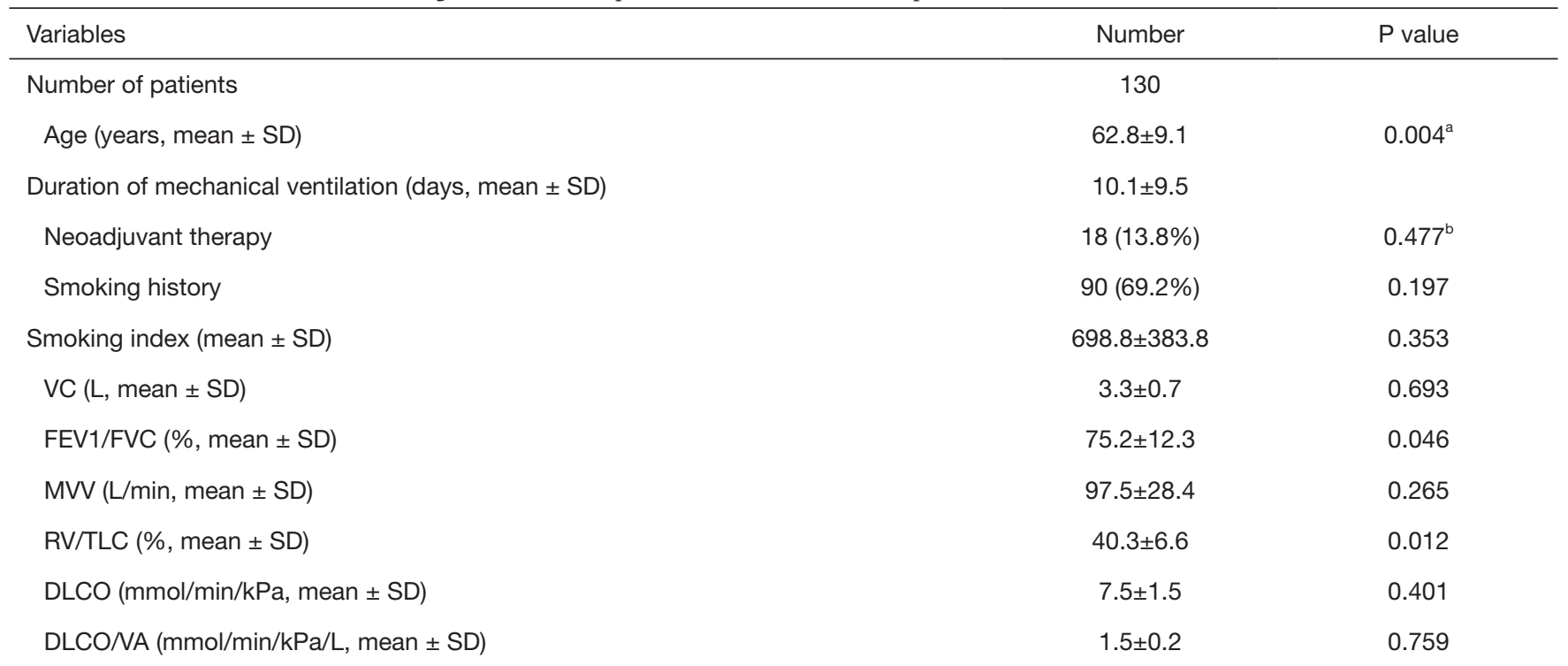

VC, vital capacity; $F E V_{1}$, forced expiratory capacity in 1 second; FVC, forced vital capacity; MVV, maximal voluntary ventilation; RV, residual volume; TLC, total lung capacity; DLCO, carbon monoxide diffusing capacity; DLCO/VA, transfer factor per unit alveolar volume; ${ }^{a}$, by Spearman's rank correlation test; ${ }^{\mathrm{b}}$, by Mann-Whitney $\mathrm{U}$ test.

As shown in Table 1, in this study, the age of patients ranged from 45 to 82 years. The average age was 62.8 years old. And the number of patients over 70 years old was 30 (23.1\%). Statistical analysis indicated that age could significantly affect the duration of mechanical ventilation ( $\mathrm{P}=0.004)$.

A total of 18 patients (13.8\%) received neoadjuvant therapy before surgery. The statistical results showed that neoadjuvant therapy did not prolong the duration of mechanical ventilation $(\mathrm{P}=0.477)$.

A total of 90 patients had a history of smoking, and statistical results suggested that smoking history as well as smoking index had no significant correlation with the patient's mechanical ventilation time $(\mathrm{P}=0.197, \mathrm{P}=0.353$, respectively).

We studied a series of parameters of lung function tests, as shown in Table 1. We found that vital capacity (VC), maximal voluntary ventilation (MVV), diffusing capacity of the lung for carbon monoxide (DLCO), diffusing capacity of the lung for carbon monoxide divided by alveolar volume (DLCO/VA) had no significant effect on the duration of mechanical ventilation. However, the decrease of forced expiratory volume in 1 second $\left(\mathrm{FEV}_{1}\right)$ /forced volume vital 
Table 2 Clinical laboratory parameters of the patients and their relationship with the duration of mechanical ventilation

\begin{tabular}{lcc}
\hline Variables & Number & P value \\
\hline White blood cell count $\left(\times 10^{9} / \mathrm{L}\right.$, mean $\left.\pm \mathrm{SD}\right)$ & $15.5 \pm 5.0$ & $0.125^{\mathrm{a}}$ \\
Thrombocytopenia, $\mathrm{n}(\%)$ & $19(14.6 \%)$ & $0.000^{\mathrm{b}}$ \\
Creatinine $(\mu \mathrm{mol} / \mathrm{L}$, mean $\pm \mathrm{SD})$ & $85.9 \pm 47.7$ & $0.000^{\mathrm{a}}$ \\
C-reactive protein $(\mathrm{mg} / \mathrm{L}$, mean $\pm \mathrm{SD})$ & $222.7 \pm 83.8$ & $0.020^{\mathrm{a}}$ \\
Lactate $(\mathrm{mmol} / \mathrm{L}$, mean $\pm \mathrm{SD})$ & $2.1 \pm 2.0$ & $0.002^{\mathrm{a}}$ \\
\hline
\end{tabular}

${ }^{\text {a }}$, by Spearman's rank correlation test; ${ }^{\mathrm{b}}$, by Mann-Whitney $\mathrm{U}$ test.

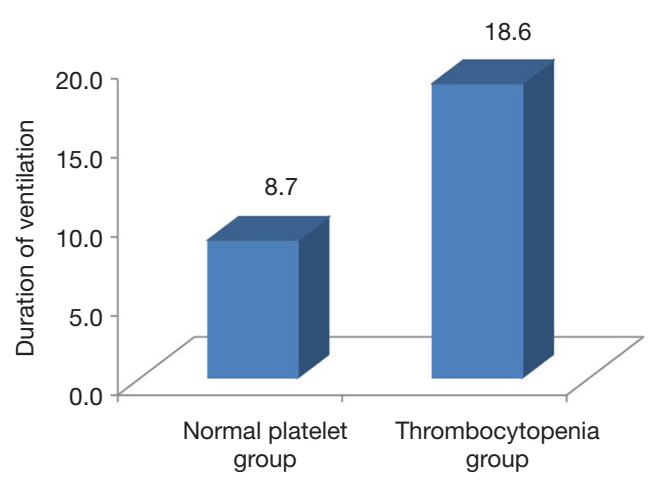

Figure 2 Comparison of mechanical ventilation duration in patients with normal platelet count and thrombocytopenia.

capacity (FVC) ratio and the increase of residual volume (RV)/total lung capacity TLC ratio can significantly prolong the duration of mechanical ventilation $(\mathrm{P}=0.046, \mathrm{P}=0.012$, respectively).

We also studied some biochemical indicators to discover their effects on the recovery of respiratory failure, as shown in Table 2. The patients' WBC count in this study ranged from 5.8 to $36.7\left(\times 10^{9} / \mathrm{L}\right)$. A total of $120(92.3 \%)$ patients had higher values than normal, the average value was 15.5 $\left(\times 10^{9} / \mathrm{L}\right)$. Data analysis revealed no correlation between WBC count and mechanical ventilation duration of the patients $(\mathrm{P}=0.125)$.

Of the total 130 patients, 19 developed thrombocytopenia. As shown in Figure 2, for patients with normal platelets count, the average duration of mechanical ventilation was 8.7 days, while for patients with thrombocytopenia, the average duration of mechanical ventilation was 18.6 days, the latter was more than twice as much as the former $(\mathrm{P}<0.001)$. It could be seen that thrombocytopenia had a significant impact on the rehabilitation of patients with respiratory failure. Once

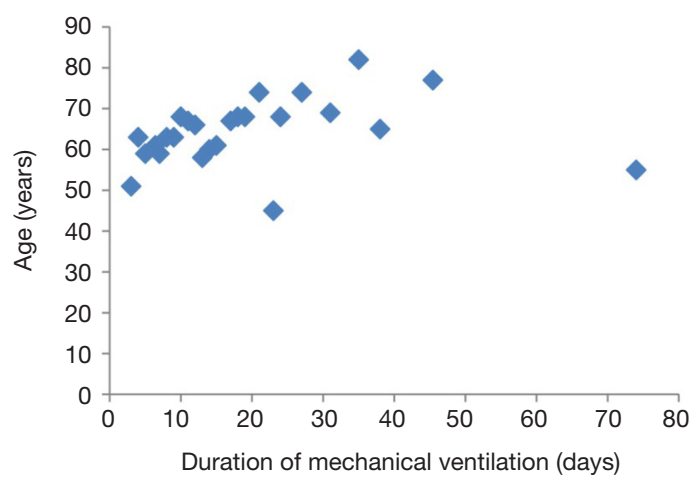

Figure 3 The relationship between age and duration of mechanical ventilation.

thrombocytopenia occurred, the duration of mechanical ventilation was remarkably prolonged.

The serum creatinine values of patients in this study ranged from 39.0 to $298.2 \mu \mathrm{mol} / \mathrm{L}$, the average value was $85.9 \mu \mathrm{mol} / \mathrm{L}$. In this study, $17(13.1 \%)$ patients had higher creatinine levels than normal $(18-110 \mu \mathrm{mol} / \mathrm{L})$. The result of statistical analysis suggested that the patients' creatinine level had an influence on the duration of mechanical ventilation $(\mathrm{P}<0.001)$.

The CRP values of patients in this study ranged from 20.7 to $465.4 \mathrm{mg} / \mathrm{L}$. Their average value was $222.7 \mathrm{mg} / \mathrm{L}$. All patients in this study had higher levels of CRP than normal $(0-8 \mathrm{mg} / \mathrm{L})$. Statistical analysis indicated that the level of CRP could influence the length of mechanical ventilation $(\mathrm{P}=0.020)$.

The lactate value in this study ranged from 0.6 to $15.0(\mathrm{mmol} / \mathrm{L})$, among them, 23 patients $(17.7 \%)$ had higher lactate levels than normal. Data analysis showed that the level of lactate could significantly affect the duration of mechanical ventilation in patients $(\mathrm{P}=0.002)$.

The scatter plots of the relationship between the factors 


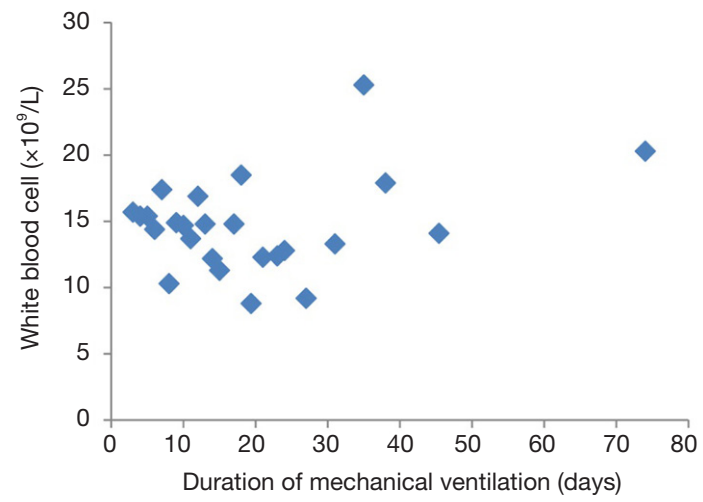

Figure 4 The relationship between white blood cell count and duration of mechanical ventilation.

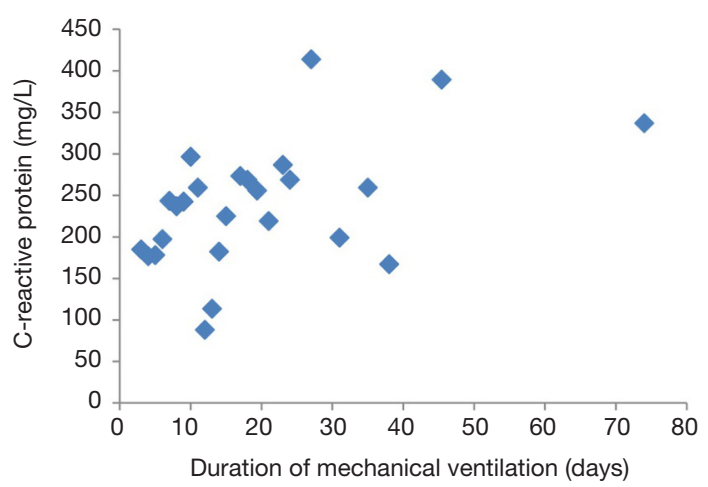

Figure 5 The relationship between serum C-reactive protein level and duration of mechanical ventilation.

studied above and the duration of mechanical ventilation were shown in Figures 3-7.

\section{Discussion}

Considering that most patients in this study recovered within one to two weeks. As we know, respiratory failure after esophageal cancer surgery is often a kind of ARDS caused by surgical trauma and inflammatory stimulation. Since the pathogenic causes are relatively simple, the prognosis may be comparable. So perhaps this type of patient could serve as a model for studying ARDS caused by traumatic inflammation, helping us to compare the effects of different factors on the recovery progress of respiratory failure.

Among the lung function indicators, we studied, $\mathrm{FEV}_{1} /$

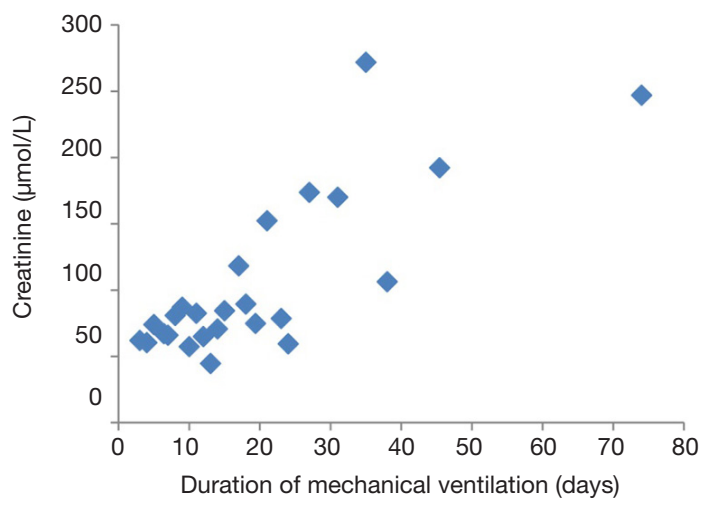

Figure 6 The relationship between serum creatinine level and duration of mechanical ventilation.

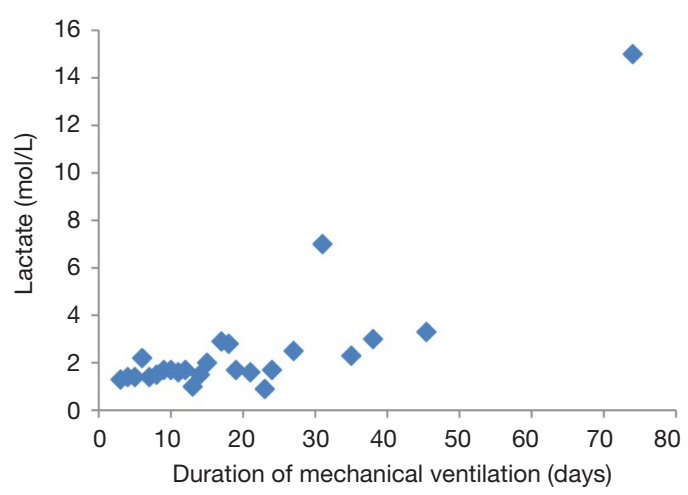

Figure 7 The relationship between serum lactate level and duration of mechanical ventilation.

FVC ratio and RV/TLC ratio were found to be risk factors to the duration of mechanical ventilation. We know that when patients' airway resistance increase, such as in patients with chronic obstructive pulmonary disease or acute attack of bronchial asthma, these two indicators will be abnormal. And when a patient has a decrease in lung elasticity, such as in emphysema, the RV/TLC ratio increases. Therefore, when the patient had some of the above abnormalities, the mechanical ventilation duration would be prolonged.

This study analyzed the influences of several factors on the recovery of respiratory failure. Owing to the decline of the physical function and the low organ reserve function of the elderly, it is often difficult for them to recover or slow to recover after illness. This study confirmed that advanced age prolonged the duration of mechanical ventilation. This reminds us that we should be more cautious in the 
treatment of elderly patients after esophageal cancer surgery.

We know that there are many factors that can cause the increase of WBC, including infection, trauma, inflammation, stress response, bleeding, etc. Therefore, the elevation of WBC is usually not clinically specific. This study also suggested that there is no statistical correlation between the values of $\mathrm{WBC}$ and the duration of mechanical ventilation. So, the level of $\mathrm{WBC}$ is less valuable in assessing the prognosis in this kind of patients.

CRP is a rapidly rising protein in plasma when the body is exposed to infection or tissue damage. As a highly sensitive indicator of acute response, plasma CRP concentration increases rapidly and significantly in trauma, infection, inflammation, surgery and acute myocardial infarction. Traditionally, CRP is considered as a nonspecific inflammatory marker. Nevertheless, the results of this study found that there was a certain correlation between CRP level and the patient's mechanical ventilation duration. In patients with higher CRP values, mechanical ventilation would take longer.

This study found that although WBC levels in routine blood tests had little effect on the prognosis of patients, thrombocytopenia was a completely different matter. Thrombocytopenia is a common clinical manifestation in severe patients. The incidence of thrombocytopenia in critically ill patients during ICU is $14 \%$ to $44 \%(9,10)$. There are many factors that could cause thrombocytopenia, including surgery, sepsis, use of vasoactive drugs, organ dysfunction, etc. $(9,11)$. This study found that thrombocytopenia had an important impact on the prognosis of patients with respiratory failure. Patients with thrombocytopenia had significantly longer ventilation duration. This suggested that thrombocytopenia could be a sensitive indicator of the severity of the disease.

Acute kidney injury (AKI) is a common critical disease, which is characterized by a rise in serum creatinine and/ or a decrease in urine volume in a short period of time. The incidence of AKI in critically ill patients is as high as $35 \%$ (12). Prospective multicenter studies have shown that severe sepsis is the leading cause of AKI $(13,14)$. In this study, a total of 17 patients (13.1\%) developed creatinine elevation. Statistical analysis showed that creatinine level was correlated with the duration of mechanical ventilation. Therefore, creatinine level can be used as an evaluation factor for the prognosis of respiratory failure after esophageal cancer surgery.

Lactate is the product of anaerobic metabolism of cells.
Arterial blood lactate elevation is an early sensitive indicator of deficiency of tissue perfusion and oxygen supply (15). It is suggested that when the level of blood lactate exceeds $4 \mathrm{mmol} / \mathrm{L}$ in sepsis patients, the mortality rate increases greatly (16). Blood lactate level may be one of the clinical indicators to evaluate the severity and prognosis of sepsis patients (17). This study found that blood lactate level was related to the recovery of patients with respiratory failure. The higher the lactate value, the longer the patient's mechanical ventilation would be. This suggested that lactate was also one of the indicators to estimate the prognosis of respiratory failure after esophagectomy.

In conclusion, this study investigated several factors that may affect the recovery of patients with respiratory failure after esophageal cancer surgery. Abnormal $\mathrm{FEV}_{1} / \mathrm{FVC}$ ratio and RV/TLC ratio as well as old age, thrombocytopenia, elevated creatinine, CRP and blood lactate were found to be risk factors for the recovery of patients with respiratory failure after esophagectomy.

The present study was conducted on a relatively larger number of patients compared to some other studies; however, the main limitation of this study is that it utilized data from a single-center and is retrospective in design. Further studies with a prospective, multicenter design will better clarify the predictive factors for respiratory failure after esophagectomy.

\section{Acknowledgments}

Funding: None.

\section{Footnote}

Reporting Checklist: The authors have completed the STROBE reporting checklist. Available at http://dx.doi. org/10.21037/tcr-20-1326a

Data Sharing Statement: Available at http://dx.doi. org/10.21037/tcr-20-1326a

Conflicts of Interest: All authors have completed the ICMJE uniform disclosure form (available at http://dx.doi. org/10.21037/tcr-20-1326a). The authors have no conflicts of interest to declare.

Etbical Statement: The authors are accountable for all aspects of the work in ensuring that questions related to the accuracy or integrity of any part of the work are 
appropriately investigated and resolved. The study was conducted in accordance with the Declaration of Helsinki (as revised in 2013). The study was approved by Ethics Committee of Sun Yat-sen University Cancer Center of B2019-028-01 and informed consent was taken from all the patients.

Open Access Statement: This is an Open Access article distributed in accordance with the Creative Commons Attribution-NonCommercial-NoDerivs 4.0 International License (CC BY-NC-ND 4.0), which permits the noncommercial replication and distribution of the article with the strict proviso that no changes or edits are made and the original work is properly cited (including links to both the formal publication through the relevant DOI and the license). See: https://creativecommons.org/licenses/by-nc-nd/4.0/.

\section{References}

1. Jemal A, Bray F, Center MM, et al. Global cancer statistics. CA Cancer J Clin 2011;61:69-90.

2. Jin D, Yao L, Yu J, et al. Robotic-assisted minimally invasive esophagectomy versus the conventional minimally invasive one: A meta-analysis and systematic review. Int J Med Robot 2019;15:e1988.

3. Booka E, Takeuchi H, Kikuchi H, et al. Recent advances in thoracoscopic esophagectomy for esophageal cancer. Asian J Endosc Surg 2019;12:19-29.

4. Takahashi C, Shridhar R, Huston J, et al. Esophagectomy from then to now. J Gastrointest Oncol 2018;9:903-9.

5. Schieman C, Wigle DA, Deschamps C, et al. Patterns of operative mortality following esophagectomy. Dis Esophagus 2012;25:645-51.

6. Jamieson GG, Mathew G, Ludemann R, et al. Postoperative mortality following oesophagectomy and problems in reporting its rate. Br J Surg 2004;91:943-7.

7. Booka E, Takeuchi H, Nishi T, et al. The Impact

Cite this article as: $\mathrm{Su} \mathrm{Q}, \mathrm{Li} \mathrm{H}$, Yan H, Wei W, Liao W, Ma G. Prognostic risk factors for respiratory failure after esophagectomy. Transl Cancer Res 2020;9(10):6362-6368. doi: $10.21037 /$ tcr-20-1326a of Postoperative Complications on Survivals After Esophagectomy for Esophageal Cancer. Medicine (Baltimore) 2015;94:e1369.

8. Yang WG, Yang C, Nie WS, et al. Perioperative management of esophageal carcinoma patients with respiratory dysfunction. Chinese Journal of Clinical Thoracic and Cardiovascular Surgery 2011;18:273-5.

9. Hui P, Cook DJ, Lim W, et al. The frequency and clinical significance of thrombocytopenia complicating critical illness: a systematic review. Chest 2011;139:271-8.

10. Williamson DR, Lesur O, Tétrault JP, et al. Thrombocytopenia in the critically ill: prevalence, incidence, risk factors, and clinical outcomes. Can J Anaesth 2013;60:641-51.

11. Williamson DR, Albert M, Heels-Ansdell D, et al. Thrombocytopenia in critically ill patients receiving thromboprophylaxis: frequency, risk factors, and outcomes. Chest 2013;144:1207-15.

12. Lameire NH, Bagga A, Cruz D, et al. Acute kidney injury: an increasing global concern. Lancet 2013;382:170-9.

13. Bagshaw SM, Uchino S, Bellomo R, et al. Septic acute kidney injury in critically ill patients: clinical characteristics and outcomes. Clin J Am Soc Nephrol 2007;2:431-9.

14. Wen Y, Jiang L, Xu Y, et al. Prevalence, risk factors, clinical course, and outcome of acute kidney injury in Chinese intensive care units: a prospective cohort study. Chin Med J (Engl) 2013;126:4409-16.

15. Tisherman SA, Barie P, Bokhari F, et al. Clinical practice guideline: endpoints of resuscitation. J Trauma 2004;57:898-912.

16. Nguyen HB, Loomba M, Yang JJ, et al. Early lactate clearance is associated with biomarkers of inflammation, coagulation, apoptosis, organ dysfunction and mortality in severe sepsis and septic shock. J Inflamm (Lond) 2010;7:6.

17. Okorie ON, Dellinger P. Lactate: biomarker and potential therapeutic target. Crit Care Clin 2011;27:299-326. 